\title{
Anterior cingulate implants for tinnitus: report of 2 cases
}

\author{
Dirk De Ridder, MD, PhD, ${ }^{1}$ Kathleen Joos, MD, $\mathrm{PhD},{ }^{2}$ and Sven Vanneste, $\mathrm{PhD}{ }^{3}$ \\ 1Department of Surgical Sciences, Section of Neurosurgery, Dunedin School of Medicine, University of Otago, New Zealand; \\ 2Department of Neurosurgery, University Hospital Antwerp, Belgium; and ${ }^{3}$ Lab for Auditory \& Integrative Neuroscience, School of \\ Behavioral and Brain Sciences, University of Texas at Dallas, Texas
}

\begin{abstract}
Tinnitus can be distressful, and tinnitus distress has been linked to increased beta oscillatory activity in the dorsal anterior cingulate cortex (dACC). The amount of distress is linked to alpha activity in the medial temporal lobe (amygdala and parahippocampal area), as well as the subgenual (sg)ACC and insula, and the functional connectivity between the parahippocampal area and the sgACC at 10 and $11.5 \mathrm{~Hz}$.

The authors describe 2 patients with very severely distressing intractable tinnitus who underwent transcranial magnetic stimulation (TMS) with a double-cone coil targeting the dACC and subsequent implantation of electrodes on the dACC. One of the patients responded to the implant and one did not, even though phenomenologically they both expressed the same tinnitus loudness and distress.

The responder has remained dramatically improved for more than 2 years with $6-\mathrm{Hz}$ burst stimulation of the dACC. The 2 patients differed in functional connectivity between the area of the implant and a tinnitus network consisting of the parahippocampal area as well as the sgACC and insula; that is, the responder had increased functional connectivity between these areas, whereas the nonresponder had decreased functional connectivity between these areas. Only the patient with increased functional connectivity linked to the target area of repetitive TMS or implantation might transmit the stimulation current to the entire tinnitus network and thus clinically improve.
\end{abstract}

http://thejns.org/doi/abs/10.3171/2015.3.JNS142880

KEY WORDS tinnitus; homeostatic emotion; anterior cingulate cortex; functional neurosurgery

$\mathrm{N}$ ONPULSATILE tinnitus is the perception of a sound in the absence of an external source and is therefore often considered a phantom sound..$^{30}$ It is related to abnormal activity in the auditory and nonauditory brain areas, ${ }^{48}$ which can be altered by neuromodulation techniques. ${ }^{24}$

As tinnitus is most commonly related to auditory deafferentation ${ }^{20}$ with $^{30,31}$ or without ${ }^{59}$ audiometric hearing loss, it has been regarded as maladaptive auditory memory traces $^{5}$ attempting to reduce the inherent auditory uncertainty associated with auditory deafferentation. ${ }^{11}$ Apart from abnormal activity, pathological functional connectivity is also associated with the presence of tinnitus as demonstrated by electroencephalography (EEG), ${ }^{57}$ magnetoencephalography (MEG), ${ }^{38,39}$ and functional MRI (fMRI). ${ }^{26,27}$ Thus, it has been proposed that the phenomenologically unified percept of tinnitus can be considered an emergent property of multiple, parallel, dynamically changing, and partially overlapping subnetworks, each with a specific spontaneous oscillatory pattern and functional connectivity signature. ${ }^{17}$ Functional imaging studies using source-localized EEG and MEG have shown that tinnitus distress is related to a network involving the anterior cingulate cortex (ACC), anterior insula, and parahippocampal area, ${ }^{6,22,56}$ whereas the auditory cortex is involved in the perceived tinnitus intensity ${ }^{44}$ The distress subnetwork is predominantly lateralized to the right, in contrast to a similar network encoding depression that is lateralized to the left. ${ }^{21}$ The loudness and distress networks are clearly separable even though they interact. Communication between these different subnetworks is proposed to occur at hubs, brain areas that are involved in multiple subnetworks simultaneously. These hubs can take part in each separable subnetwork at different frequencies. Com-

ABBREVIATIONS ACC = anterior cingulate cortex; BA = Brodmann area; BOLD = blood oxygen level-dependent; $d=$ dorsal; $D L P F C=$ dorsolateral prefrontal cortex; EEG = electroencephalography; $\mathrm{fMRI}=$ functional MRI; HADS = Hospital Anxiety Depression Scale; $M E G$ = magnetoencephalography; NRS = numeric rating scale; $r$ = repetitive; $\mathrm{sg}=$ subgenual; sLORETA = standardized low-resolution electromagnetic tomography; tDCS = transcranial direct current stimulation; TENS = transcutaneous electrical nerve stimulation; TMS = transcranial magnetic stimulation; $\mathrm{TQ}=$ tinnitus questionnaire.

SUBMITTED December 22, 2014. ACCEPTED March 12, 2015.

INCLUDE WHEN CITING Published online August 28, 2015; DOI: 10.3171/2015.3.JNS142880. 
munication between the subnetworks is proposed to occur at discrete oscillatory frequencies in healthy controls. ${ }^{3}$ In tinnitus the amount of distress present is related to 10- and $11.5-\mathrm{Hz}$ oscillations between the parahippocampal area and the subgenual (sg)ACC. ${ }^{46}$ As such, the brain uses multiple nonspecific networks in parallel, each with their own oscillatory signature, that adapt to the context to construct a unified percept possibly by synchronized activation integrated at the hubs at discrete oscillatory frequencies. ${ }^{17}$

Whether an external auditory stimulus is consciously perceived depends on activity in the dorsal (d)ACC and insula, ${ }^{36}$ which is part of the salience network. ${ }^{40}$ The dACC and insula activation in tinnitus can thus be considered as a paradoxical salience attributed to a nonmeaningful internally generated sound, which may thereby remain conscious. Thus, modulating the dACC could have an effect both on the loudness of the tinnitus and on the distress and mood, as demonstrated by transcranial magnetic stimulation (TMS) with a double-cone coil targeting this area $^{51,53,54}$ The problem with the repetitive (r)TMS sessions is that the clinical effect is short-lived, which could be alleviated by implanting an electrode on the AACC for the permanent suppression of increased tinnitus-related dACC hyperactivity.

After obtaining approval from the ethics committee and informed consent from the patients, we implanted bilateral electrodes on the dACC for tinnitus suppression in 2 patients, both of whom also underwent rTMS with the double-cone coil in a placebo-controlled way.

\section{Case Reports \\ Case 1}

History and Examination

A 64-year-old man presented to the multidisciplinary Tinnitus Research Initiative Clinic at the $\mathrm{BRAI}^{2} \mathrm{~N}$ Neu- romodulation Center at Antwerp University, Belgium. He suffered from bilateral tinnitus, which suddenly arose overnight but had persisted for 39 years. The tinnitus was perceived as a pure tone, somewhat more pronounced on the left than the right. It was scored 10/10 for both loudness and distress on a numeric rating scale (NRS), and increased with fatigue and stress as well as with noise exposure. He also suffered from hyperacusis (sound intolerance) associated with high-frequency hearing loss (Fig. 1 upper) compatible with presbycusis.

The patient denied having headaches or any neck pain that modulated the tinnitus. There were no other signs of somatosensory modulation of his tinnitus. He experienced no overt or covert hemifacial spasms or geniculate neuralgia. He had some balance disorders in the form of a drunken feeling with a slight deviation to the right. Tinnitus matching demonstrated that the tinnitus was centered at $4000 \mathrm{~Hz}, 10 \mathrm{~dB}$ above the hearing threshold, and was associated with high-pitched hearing loss. On his tinnitus questionnaire (TQ) ${ }^{19}$ the patient scored 68, that is, Grade 4, psychologically decompensated tinnitus. His Hospital Anxiety Depression Scale (HADS) scores were 13 each for anxiety and depression.

Brain CT was unremarkable, and MRI showed multiple white matter lesions, which may or may not have been related to the patient's symptoms. ${ }^{32}$ Resting state sourcelocalized standardized low-resolution electromagnetic tomography (sLORETA) ${ }^{35}$ EEG was performed to visualize abnormal brain activity, which could be used as a target for noninvasive neuromodulation. Treatment with flupentixol plus melitracen (Deanxit) and clonazepam ${ }^{29}$ did not benefit the patient, nor did cyclobenzaprine, ${ }^{52}$ naltrexone, ${ }^{45}$ or acamprosate. ${ }^{1}$ A small improvement on the right side was noted by wearing a combination hearing aid and noise masker to a score of $8 / 10$ for loudness but $10 / 10$ for distress on an NRS.

Since the patient's tinnitus was intractable, neuro-

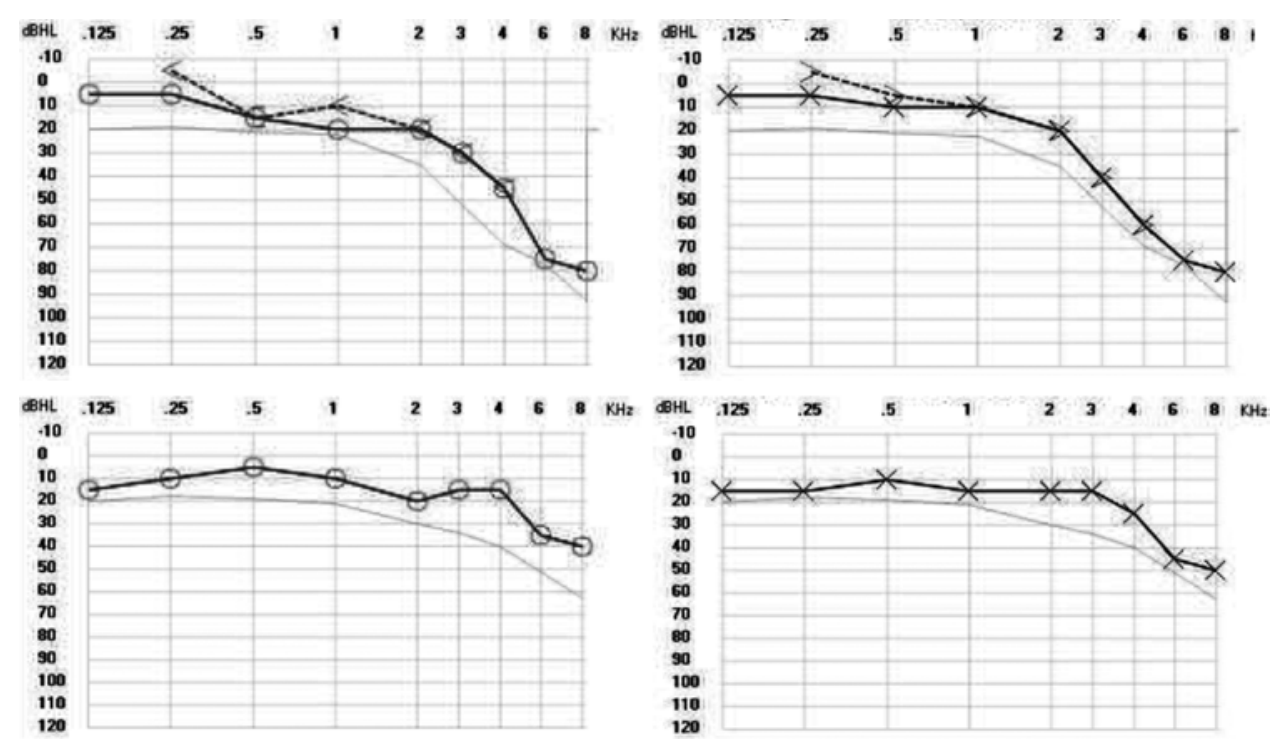

FIG. 1. Upper: Case 1. Audiometry results showing bilateral high-frequency hearing loss, that is, presbycusis, normal for the patient's age. Lower: Case 2. Audiometry results demonstrating limited high-frequency hearing loss, which can be considered normal hearing for the patient's age. Gray line represents normal hearing for the patient's age group; solid line, air conduction; dashed line, bone conduction. Right side (left graph), left side (right graph). 
modulation trials were proposed, consisting of TMS of the auditory cortex,$^{18}$ bifrontal transcranial direct current stimulation (tDCS) of the dorsolateral prefrontal cortex (DLPFC) ${ }^{49}$ and transcutaneous electrical nerve stimulation (TENS) of the C-2 nerve..$^{55}$ None of these approaches yielded any benefit for the patient.

Therefore, fMRI was performed according to a protocol previously described ${ }^{4,41}$ but adjusted to the patient's tinnitus-matched frequency. ${ }^{12}$ Pathological blood oxygen level-dependent (BOLD) activation could then be used as a target for neuromodulation using a neuronavigation system. ${ }^{4,14}$ Pathological activity was indicated by the BOLD activation elicited by a $4000-\mathrm{Hz}$ sound presentation (the patient's tinnitus frequency) and not by a control frequency (1000 Hz; Fig. 2 upper).

Transcranial magnetic stimulation at $1 \mathrm{~Hz}$ and an intensity of $90 \%$ of the motor threshold directed to a BOLD fMRI activation area at the right DLPFC yielded a transient $50 \%$ decrease in subjectively perceived tinnitus loudness on the right, but not on the left, whereas placebo TMS had no effect. This result was confirmed by placebocontrolled burst rTMS of the right DLPFC at $50 \%$ of the machine output. Transcranial magnetic stimulation at the ventrolateral PFC at $10 \mathrm{~Hz}$ yielded no beneficial effect, whereas 1-Hz TMS of the right angular gyrus (Brodmann area [BA]39) only improved the tinnitus by 1 point on the NRS. Results were not further improved by adding tDCS to the TMS. Because TMS of the right DLPFC yielded the best transient response, 10 sessions (daily for 2 weeks) of rTMS targeting the right DLPFC were performed. Unfortunately, this had only a transient and marginal effect. For a period of 2 weeks, it improved the patient's tinnitus loudness on the right from $8 / 10$ to $5 / 10$, but his left-sided tinnitus remained at 10/10; his distress improved from 9/10 to $7 / 10$ on an NRS. However, questionnaires evaluating his mood and distress remained unaltered: the TQ changed from 68 to 67, and his HADS scores changed from 13 to 12 for anxiety and from 13 to 11 for depression.

Since part of the anterior cingulate gyrus in the patient showed BOLD activation as well, TMS at $5 \mathrm{~Hz}$ with the
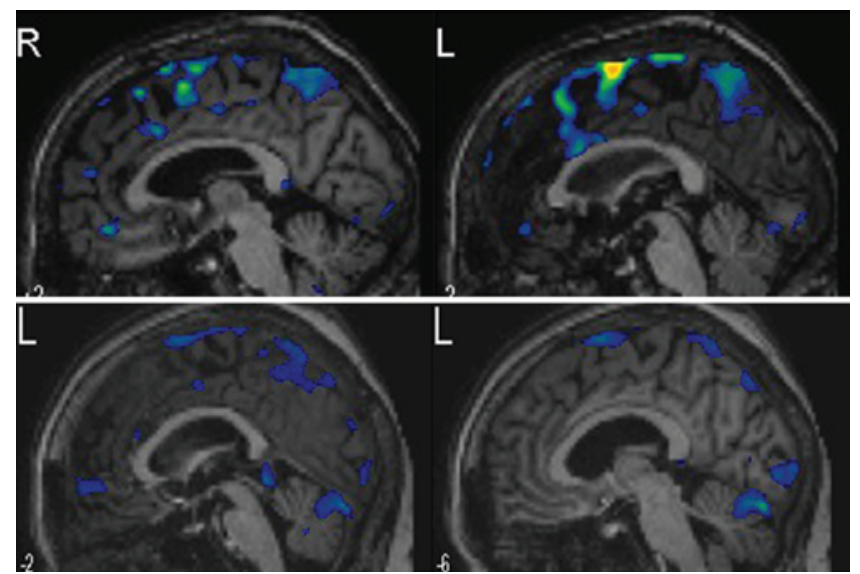

FIG. 2. Upper: Case 1. Functional MR images demonstrate a BOLD signal at the dACC evoked by a tinnitus-matched frequency presentation in the scanner. Lower: Case 2. A small BOLD activation in the rostral and dorsal anterior cingulate cortex evoked by a tinnitus-matched frequency presentation in the scanner. Figure is available in color online only. double-cone coil was performed in both tonic and burst mode. In the tonic mode at $1 \mathrm{~Hz}$, the tinnitus improved to $5 / 10$ on the right and $7 / 10$ on the left; at $5 \mathrm{~Hz}$, it improved to $2 / 10$ on the right and $6 / 10$ on the left; and at $10 \mathrm{~Hz}$, it improved to $3 / 10$ on the right and 6/10 on the left. With theta-burst TMS at $5 \mathrm{~Hz}$, the tinnitus was reduced to $2 / 10$ on the right and 5/10 on the left. Placebo TMS did not yield any benefit. Transcranial magnetic stimulation with the double-cone coils was repeated, and similar transient improvements were obtained. Therefore, rTMS consisting of 10 sessions (daily for 2 weeks) was proposed. Unfortunately, this did not result in long-lasting improvement. As the double-cone TMS targeting of the dACC resulted in better tinnitus suppression than the DLPFC TMS, the dACC was selected for implantation (the DLPFC has been previously targeted for tinnitus suppression ${ }^{14}$ ).

\section{Operation}

We performed an open neurosurgical approach consisting of a small right-sided frontal craniotomy for a transfalcine approach, inserting 2 electrodes for bilateral dACC stimulation. After the induction of anesthesia, intubation, and ventilation, the patient was fixed in a Mayfield headrest in a supine position, his neck slightly flexed with $0^{\circ}$ rotation. After the registration of preoperatively administered skin fiducial markers for neuronavigation with the Stealth frameless stereotactic system, the patient's head was disinfected and draped in a sterile fashion. A laterolateral frontal incision was made within the hairline crossing the midline, followed by a $4 \times 4-\mathrm{cm}$ right frontal craniotomy crossing the superior sagittal sinus. Subsequently, the dura mater was incised in a U-shaped fashion and was reflected across the midline. This was followed by a neuronavigated approach between the right frontal lobe and the falx (Fig. 3). Once the target was localized, the falx was incised and 2 Lamitrode 44 electrodes (St. Jude Medical, Neurodivision) were sutured back to back and inserted, with the fMRI BOLD activity as the target (Fig. 2 upper). The electrodes were sutured to the falx, the operative site was thoroughly rinsed, the dura was closed in a primary fashion, and the bone was repositioned and fixed. The skin was closed, and the patient was removed from the headrest and woken up from the anesthesia.

\section{Postoperative Course}

On the 1st postoperative day, the electrodes were activated in the $6-\mathrm{Hz}$ tonic mode. The electrode pole configuration was +-+-+-+- (that is, alternating anodes and cathodes) to generate a large stimulation field. On the 2nd postoperative day, the patient was discharged. The $6 \mathrm{~Hz}$ was selected as a frequency similar to the one $(5 \mathrm{~Hz})$ that yielded the best results with TMS.

After 1 week the patient was evaluated, and his clinical state was dramatically improved. All measures were basically halved in severity. Distress was reduced from $9 / 10$ to $5 / 10$, or from Grade 4 (very severe tinnitus, psychologically decompensated) to Grade 2 tinnitus (moderate). His subjective loudness perception was decreased from 10/10 to $5 / 10$ in the right ear and from $8 / 10$ to $4 / 10$ in the left ear. Moreover, the percentage of time that the tinnitus was dominantly present was halved from $100 \%$ to $50 \%$. After 

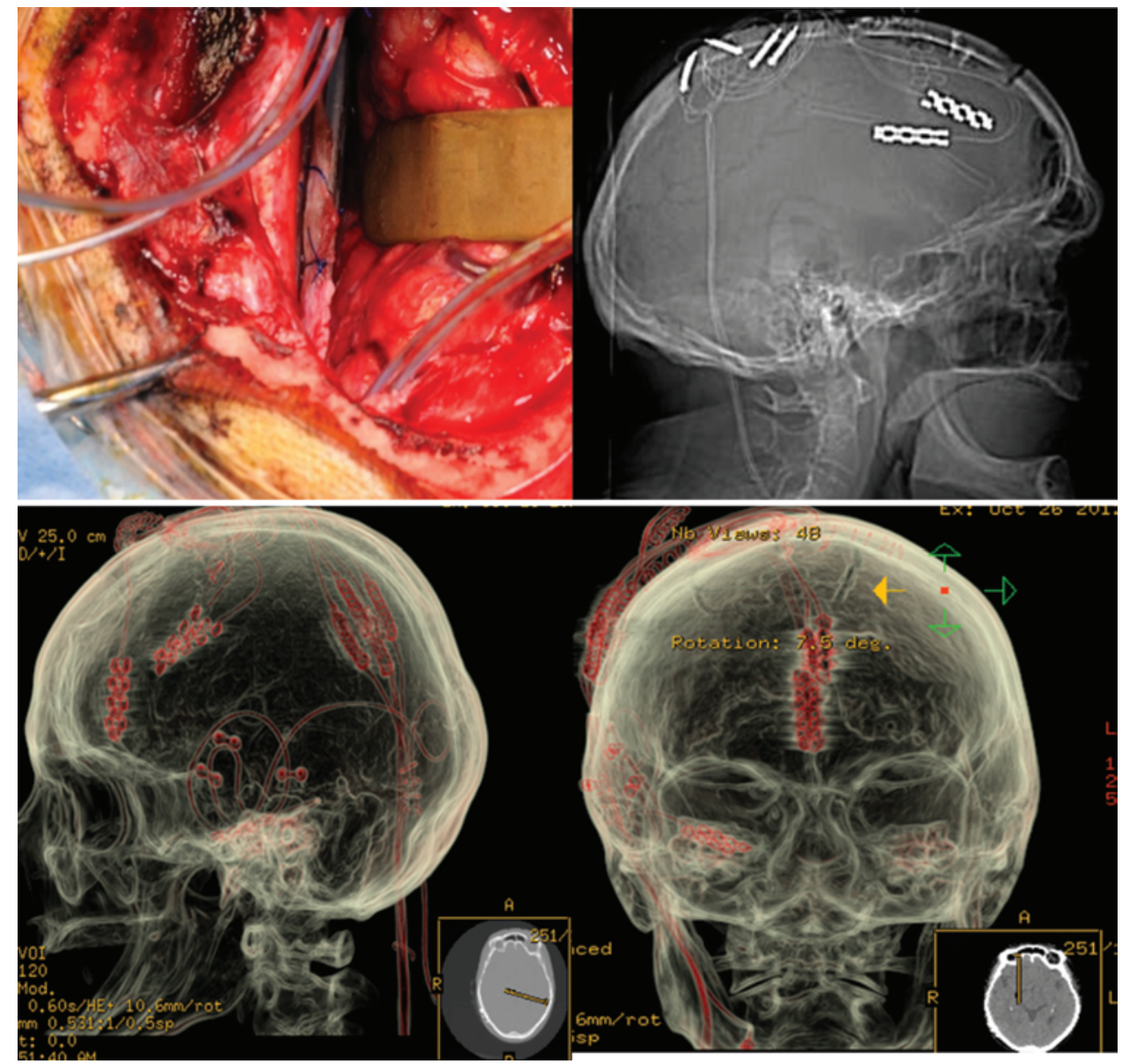

FIG. 3. Case 1. Upper: Implant of back-to-back electrode on the dACC and supplementary motor area. Lower: Implant on dACC and pregenual ACC with back-to-back leads for bilateral cingulate stimulation. Figure is available in color online only.

4 weeks, his stimulation design was altered to verify if further improvement could be obtained. A recently developed stimulation design consisting of a $6-\mathrm{Hz}$ burst mode, 5 spikes at a $500-\mathrm{Hz}$ spike mode, and a $1000-\mu$ sec pulse width at $1.4 \mathrm{~mA}^{15,16}$ was used in an attempt to improve his clinical picture. Burst stimulation was provided by an Eon implantable pulse generator (St. Jude Medical, Neurodivision) programmed with a custom-made device. And indeed, his distress was further reduced to $3 / 10$, remaining moderate; his subjectively perceived loudness was further reduced to $3 / 10$ on the left (his worst ear) and remained at $4 / 10$ on the right; and his anxiety and depression scores improved from 13 to 6 and from 13 to 8 , respectively.

Other burst frequencies were also tested: $2,4,8$, and 10 $\mathrm{Hz}$, but the $6-\mathrm{Hz}$ burst mode remained clinically superior and was therefore maintained. The patient's beneficial effect has remained for 2 years.

\section{Case 2}

\section{History and Examination}

A 63-year-old woman presented to the multidisciplinary Tinnitus Research Initiative Clinic at the BRAI²N Neuromodulation Center at Antwerp University, Belgium. She suffered from left-sided tinnitus, which had developed 6 years prior to the consultation. The tinnitus was perceived as a narrow band noise, was scored 10/10 for both loudness and distress on an NRS, and increased on fatigue and stress as well as with noise exposure. She also suffered from dizziness, sometimes associated with vomiting. She denied having headaches and had no signs of somatosensory modulation of her tinnitus. She experienced no overt or covert hemifacial spasms or geniculate neuralgia.

Tinnitus matching demonstrated that the tinnitus was centered around $5000 \mathrm{~Hz}, 15 \mathrm{~dB}$ above the hearing threshold, and was associated with high-pitched hearing loss (Fig. 1 lower). On her TQ,$^{19}$ she scored 71, that is, Grade 4, psychologically decompensated tinnitus. Her HADS scores for anxiety and depression were 15 and 13, respectively.

Brain CT and MRI were unremarkable. Resting state source-localized SLORETA EEG was performed to visualize abnormal brain activity, which could be used as a target for noninvasive neuromodulation.

She had taken citalopram, flupentixol plus melitracen, valproic acid, quetiapine, lorazepam, trazodone, and ranitidine, all to no avail. At an earlier stage she had undergone a right-sided intradural subtemporal implant targeting the hippocampal area without success (Fig. 2 lower). ${ }^{13}$

Since her tinnitus was intractable, neuromodulation trials were proposed, consisting of TMS of the right auditory cortex in the 1-, 3-, 5-, and $10-\mathrm{Hz}$ tonic mode ${ }^{18}$ as well as in 
the theta-, alpha-, and beta-burst mode. ${ }^{7,8}$ Bifrontal tDCS ${ }^{49}$ and TENS of the C-2 nerve at different frequencies did not bring any benefit. ${ }^{55}$

Because of the treatment failure, fMRI was requested according to a protocol previously described ${ }^{4,41}$ but adjusted to the patient's tinnitus-matched frequency. ${ }^{12}$ Pathological BOLD activation could then be used as a target for neuromodulation using a neuronavigation system. ${ }^{4,14}$ Pathological activity was indicated by the BOLD activation elicited by a $5000-\mathrm{Hz}$ sound presentation (the patient's tinnitus frequency inside the scanner) and not by a control frequency $(1000 \mathrm{~Hz})$.

Transcranial magnetic stimulation at 1 and $10 \mathrm{~Hz}$ and an intensity of $90 \%$ of the motor threshold directed to a BOLD fMRI activation area at the right DLPFC, BA39 left, BA39 right, BA40, and the auditory cortex yielded no improvement whatsoever, and neither did placebo TMS. The same areas (BA39, BA40, DLPFC) were targeted by bilateral tDCS and transcranial alternating current stimulation (tACS) to no avail. The TQ score changed from 71 to 72 , and her HADS scores changed from 15 to 14 for anxiety and from 13 to 12 for depression.

Neurofeedback using sLORETA was offered, training gamma down and alpha up in the parahippocampal area. After 5 sessions the patient had no benefit.

Ultimately, after the failed subdural implant targeting the hippocampal area, 3 sessions of rTMS with a doublecone coil were performed, targeting the dACC at 1,3, and $5 \mathrm{~Hz}$. They did not yield any benefit either. The only neuromodulation session that improved the tinnitus entailed a tDCS session with the cathode over the left cerebellum and the anode on the right shoulder. This reduced the tinnitus loudness from $10 / 10$ to $7 / 10$ and distress from $10 / 10$ to $8 / 10$. This effect lasted for 1 hour.

\section{Implantation}

A successful outcome for implants on the auditory cortex is not correlated with successful TMS. ${ }^{9,12}$ Thus, it is possible that the same holds true for the ACC. In other words, as there are no data demonstrating that TMS can predict outcome in implanted electrodes in tinnitus, the option of an implant on the ACC was still considered in view of the beneficial effects in the first patient.

Therefore, a bilateral dACC as well as a pregenual ACC implant was proposed, using the same technique as described above. The pregenual electrodes were added because the pregenual ACC is part of the noise-cancelling mechanism. ${ }^{13}$ But with neither tonic nor burst stimulation at different frequencies could any benefit be obtained in a systematic way.

\section{Postimplantation Course}

Two years after the implantation, the electrodes were removed due to possible infection. The tinnitus has remained unchanged.

\section{Connectivity Changes Between Cases 1 and 2}

Electroencephalography recordings were obtained in a fully lit room with the patient (Cases 1 and 2) sitting upright on a small but comfortable chair. The actual re- cording lasted approximately 5 minutes. The EEG was sampled with 19 electrodes (Fp1, Fp2, F7, F3, Fz, F4, $\mathrm{F} 8, \mathrm{~T} 7, \mathrm{C} 3, \mathrm{Cz}, \mathrm{C} 4, \mathrm{~T} 8, \mathrm{P} 7, \mathrm{P} 3, \mathrm{Pz}, \mathrm{P} 4, \mathrm{P} 8, \mathrm{O} 1, \mathrm{O} 2)$ in the standard International 10-20 placement system referencing linked ears, and impedances were checked to remain below $5 \mathrm{k} \Omega$. Data were collected with the patient's eyes closed (sampling rate $1024 \mathrm{~Hz}$, band pass $0.15-200 \mathrm{~Hz}$ ). Data were resampled to $128 \mathrm{~Hz}$, band-pass filtered (fast Fourier transform filter) to $2-44 \mathrm{~Hz}$, subsequently transposed into Eureka! Software (NovaTech EEG, Inc.), plotted, and carefully inspected for manual artifact rejection. All episodic artifacts were removed from the stream of the EEG. Average Fourier cross-spectral matrices were computed for bands delta $(2-3.5 \mathrm{~Hz})$, theta $(4-7.5 \mathrm{~Hz})$, alpha $(8-12 \mathrm{~Hz})$, beta $(13-30 \mathrm{~Hz})$, and gamma $(30.5-45 \mathrm{~Hz})$.

We used a normative database of 20 healthy subjects to compare with the 2 tinnitus patients. Exclusion criteria were known psychiatric or neurological illness, psychiatric history, drug/alcohol abuse in a participant or any relative, current psychotropic/CNS active medications, history of head injury (with loss of consciousness) or seizures, headache, and physical disability. About 3-5 minutes of EEG activity was continuously recorded while the participant, with eyes closed, sat on a comfortable chair in a quiet and dimly lit room. Electroencephalography data were acquired at the 19 standard leads prescribed by the International 10-20 system (Fp1, Fp2, F7, F3, Fz, F4, F8, T3, C3, Cz, C4, T4, T5, P3, Pz, P4, T6, O1, O2) using both earlobes as reference and enabling a $60-\mathrm{Hz}$ notch filter to suppress power line contamination. The resistance of all electrodes was kept below $5 \mathrm{k} \Omega$. We removed all biological, instrumental, environmental, and stimulation artifacts, paying particular attention to biological artifacts generated by the eyes, the heart, and the muscles of the neck, face, and jaw via independent component analysis followed by visual inspection on a high-resolution screen, and epochs containing visible artifacts were marked and ignored for ensuing analysis.

Standardized LORETA was used to estimate the intracerebral electrical sources that generated the scalp-recorded activity in each of the 7 frequency bands. Standardized LORETA computes electric neuronal activity as current density $\left(\mathrm{A} / \mathrm{m}^{2}\right)$ without assuming a predefined number of active sources. The sLORETA solution space consists of 6239 voxels (voxel size $5 \times 5 \times 5 \mathrm{~mm}$ ) and is restricted to cortical gray matter and hippocampi, as defined by the digitized Montreal Neurological Institute probability atlas. To reduce confounds that have no regional specificity, such as total power intersubject variability, a global normalization of the sLORETA images was performed prior to statistical analyses.

Statistical dependence can be estimated by measuring correlation or covariance, spectral coherence, or phase locking. Functional connectivity is often calculated between all elements of a system, regardless of whether these elements are connected by direct structural links. Unlike structural connectivity, functional connectivity is highly time dependent. Statistical patterns between neuronal elements fluctuate on multiple time scales, some as short as $10 \mathrm{~s}$ or $100 \mathrm{~s}$ of msec. It should be noted that functional connectivity does not make any explicit reference to specific 
directional effects or to an underlying structural model. Coherence and phase synchronization between time series corresponding to different spatial locations are usually interpreted as indicators of the "connectivity." However, any measure of dependence is highly contaminated with an instantaneous, nonphysiological contribution due to volume conduction. ${ }^{34}$ However, Pascual-Marqui ${ }^{33}$ introduced a new technique (that is, Hermitian covariance matrices) that removes this confounding factor. As such, this measure of dependence can be applied to any number of brain areas jointly, that is, distributed cortical networks, whose activity can be estimated with sLORETA. Measures of linear dependence (coherence) between the multivariate time series are defined. The measures are expressed as the sum of lagged dependence and instantaneous dependence. The measures are nonnegative, take the value 0 only when there is independence, and are defined in the frequency domain. Based on this principle, lagged linear connectivity was calculated. Regions of interest were defined based on previous brain research on tinnitus including the dACC, sgACC, pregenual ACC, parahippocampus, and auditory cortex.

A comparison between Cases 1 and 2 on EEG revealed some interesting results. The patient who responded to the dACC implant (Case 1) had increased alpha functional connectivity between the parahippocampal area and the sgACC in comparison with healthy controls (Fig. 4 left). The nonresponder (Case 2) had decreased functional connectivity between these areas in comparison with healthy controls (Fig. 4 right).

To have a better understanding of how the tinnitus network is involved for both, we looked at the connectivity among the dACC, pregenual ACC, parahippocampus, and auditory cortex. The patient who responded to treatment was characterized by increased functional connectivity in a network that encompasses the dACC, pregenual ACC, parahippocampal area, and auditory cortex, all areas involved in the tinnitus network in comparison with healthy controls for the alpha and beta frequency bands. The opposite was true for the nonresponder, in whom we noticed a decrease in connectivity within the tinnitus net-

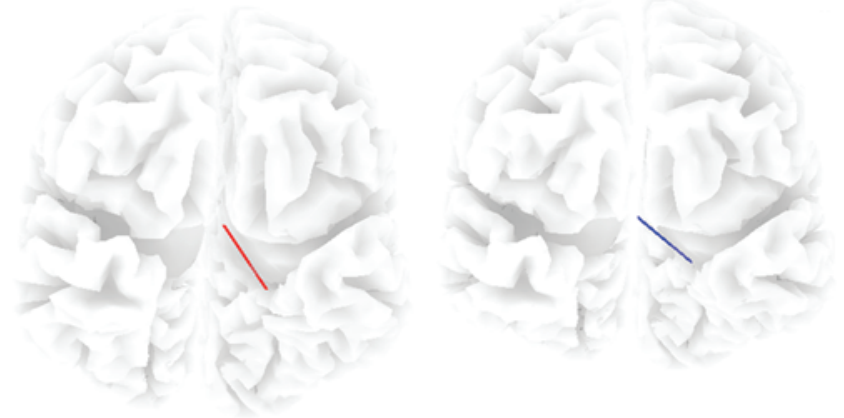

FIG. 4. Left: A comparison between the responder (Case 1) and healthy controls for high alpha frequency between the parahippocampus and the sgACC demonstrates that the responder has increased alpha functional connectivity between the sgACC and parahippocampal area (red line), known to be related to high distress. ${ }^{46}$ Right: A comparison between the nonresponder (Case 2) and healthy controls demonstrates a decrease in high alpha connectivity between the parahippocampus and the sgACC (blue line). Figure is available in color online only. work in comparison with healthy controls for the alpha and beta frequency bands. A direct comparison between the responder and nonresponder demonstrated increased connectivity among the dACC, pregenual ACC, parahippocampal area, and auditory cortex for the responder for the alpha and beta frequency bands (Fig. 5).

\section{Discussion}

Tinnitus is clinically assessed by quantifying its loudness and the impact tinnitus has on daily living, for example, by measuring tinnitus-related distress and mood changes. ${ }^{23}$ Whereas subjectively perceived loudness as measured by a visual analog scale or NRS seems to be related to auditory cortex gamma band activity, ${ }^{44}$ the presence of distress, as measured with questionnaires, is related to increased activity in the dACC and insula. ${ }^{10,43,50,56}$ However, the amount of distress is dependent on alpha activity in the parahippocampal area extending via the hippocampus to the amygdala to the $\mathrm{sgACC}$, especially by
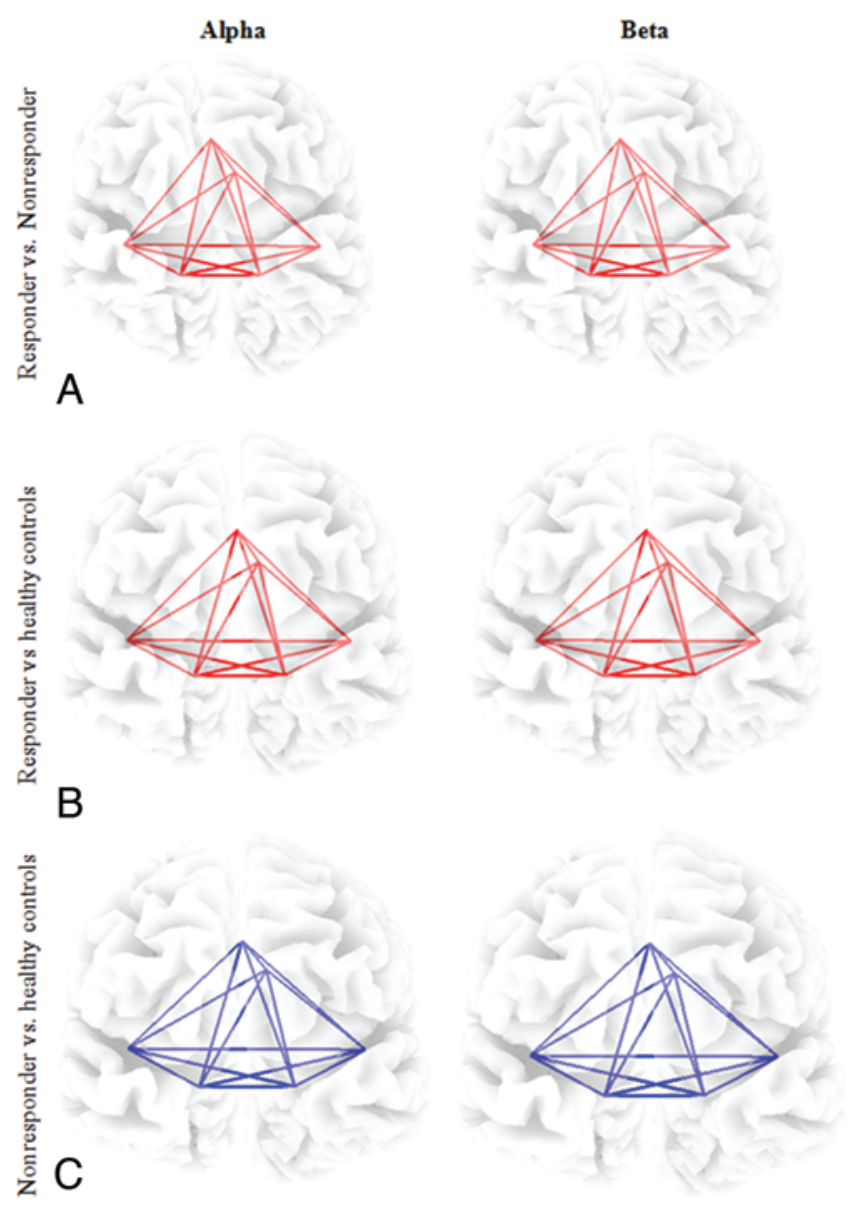

FIG. 5. A comparison between the responder and nonresponder (A) and the responder versus healthy controls $(B)$ and the nonresponder versus healthy controls $(\mathrm{C})$. The responder to the double-cone TMS and ACC implant has more functional connections between a putative tinnitus network ${ }^{17}$ and the target of neuromodulation (dACC) than the nonresponder and the healthy control group. In contrast, the nonresponder has fewer functional connections between the tinnitus network and the $\mathrm{dACC}$ than the responder and the healthy control group. Figure is available in color online only. 
functional connection at a discrete frequency $(10 \mathrm{~Hz}$ for Grade 3 and $11.5 \mathrm{~Hz}$ for Grade 4) between the parahippocampal area and the sgACC. ${ }^{46}$ These connectivity changes are confirmed on the pretreatment source-localized EEGs in this study, but there is a very striking difference between the 2 patients. Although the patient who responded to the dACC implant (Case 1) has the previously described increased alpha functional connectivity between the parahippocampal area and the sgACC, the nonresponder (Case 2) has decreased functional connectivity between these areas, even though both patients presented with very severe tinnitus.

The most salient findings from this report of 2 cases are that dACC stimulation does not work in every tinnitus patient who is highly distressed and that pretreatment EEG functional connectivity and rTMS with a double-cone coil could be predictive of the implant's success. Indeed, the dACC implant was highly successful in the patient who responded well to the double-cone coil TMS, whereas the patient who did not have any amelioration from the double-cone coil TMS targeting of the dACC did not benefit from the implant either.

Furthermore, the patient who responded to rTMS was characterized by increased functional connectivity in a network that encompasses the dACC, pregenual ACC, parahippocampal area, and auditory cortex, all areas involved in the tinnitus network ${ }^{5,17,57}$ and known to be altered by tinnitus improvement ${ }^{49}$ or worsening. ${ }^{58}$ The patient who did not respond demonstrated markedly decreased functional connectivity in this network. This is reminiscent of what has been demonstrated in auditory cortex stimulation via implanted electrodes. The success of stimulation also depends on the presence of good functional connectivity between the auditory cortex (where the electrode was placed) and the parahippocampal gyrus, ${ }^{9}$ which has been considered a critical hub in the tinnitus network, as it is involved in tinnitus in general ${ }^{27,42,57}$ and in tinnitus lateralization more specifically, ${ }^{47}$ as well as in tinnitus distress ${ }^{37,46}$ and tinnitus-related depression. ${ }^{21}$

Whereas these are only case reports, our results suggest that it is worthwhile to further explore this avenue of neuromodulation for tinnitus in severely distressed patients and that a biomarker consisting of EEG functional connectivity and/or rTMS might become important in selecting the right patient for this technique. If indeed a larger study provides evidence that EEG functional connectivity and TMS can predict who will and will not respond to an implant in the dACC, the approach used here is elegant in that any neurosurgeon can perform it. No experience in deep brain surgery with stereotactic frames is required, and the duration of the surgery is approximately 1 hour. Although a neuronavigation system might be helpful, any neurosurgeon could retrieve the dACC based on anatomical knowledge and visual landmarks.

Furthermore, it would be of interest to see whether the combination of EEG functional connectivity and TMS would be predictive in other treatments targeting the anterior cingulate gyrus, such as depression, ${ }^{28}$ pain, ${ }^{2}$ anorexia nervosa, ${ }^{25}$ or addiction (unpublished data, 2015).

The results also show that the beneficial effect seems to remain stable in time, suggesting that for intractable severely distressing tinnitus, $\mathrm{dACC}$ stimulation via direct implantation of paddle electrodes is worth further investigation and that burst pattern stimulation might be better than tonic stimulation for maintaining stable results.

\section{Acknowledgments}

We thank Silvia Kovacs and Stefan Sunaert for the fMRI.

\section{References}

1. Azevedo AA, Figueiredo RR: Tinnitus treatment with acamprosate: double-blind study. Braz J Otorhinolaryngol 71:618-623, 2005

2. Boccard SG, Fitzgerald JJ, Pereira EA, Moir L, Van Hartevelt TJ, Kringelbach ML, et al: Targeting the affective component of chronic pain: a case series of deep brain stimulation of the anterior cingulate cortex. Neurosurgery 74:628-637, 2014

3. Congedo M, John RE, De Ridder D, Prichep L, Isenhart R: On the "dependence" of "independent" group EEG sources; an EEG study on two large databases. Brain Topogr 23:134138,2010

4. De Ridder D, De Mulder G, Walsh V, Muggleton N, Sunaert S, Møller A: Magnetic and electrical stimulation of the auditory cortex for intractable tinnitus. Case report. J Neurosurg 100:560-564, 2004

5. De Ridder D, Elgoyhen AB, Romo R, Langguth B: Phantom percepts: tinnitus and pain as persisting aversive memory networks. Proc Natl Acad Sci U S A 108:8075-8080, 2011

6. De Ridder D, Fransen H, Francois O, Sunaert S, Kovacs S, Van De Heyning P: Amygdalohippocampal involvement in tinnitus and auditory memory. Acta Otolaryngol Suppl 126:50-53, 2006

7. De Ridder D, van der Loo E, Van der Kelen K, Menovsky T, van de Heyning P, Moller A: Do tonic and burst TMS modulate the lemniscal and extralemniscal system differentially? Int J Med Sci 4:242-246, 2007

8. De Ridder D, van der Loo E, Van der Kelen K, Menovsky T, van de Heyning P, Moller A: Theta, alpha and beta burst transcranial magnetic stimulation: brain modulation in tinnitus. Int J Med Sci 4:237-241, 2007

9. De Ridder D, Vanneste S: Targeting the parahippocampal area by auditory cortex stimulation in tinnitus. Brain Stimul 7:709-717, 2014

10. De Ridder D, Vanneste S, Congedo M: The distressed brain: a group blind source separation analysis on tinnitus. PLoS One 6:e24273, 2011

11. De Ridder D, Vanneste S, Freeman W: The Bayesian brain: Phantom percepts resolve sensory uncertainty. Neurosci Biobehav Rev 44:4-15, 2014

12. De Ridder D, Vanneste S, Kovacs S, Sunaert S, Menovsky T, van de Heyning P, et al: Transcranial magnetic stimulation and extradural electrodes implanted on secondary auditory cortex for tinnitus suppression. J Neurosurg 114:903-911, 2011

13. De Ridder D, Vanneste S, Menovsky T, Langguth B: Surgical brain modulation for tinnitus: the past, present and future. $\mathbf{J}$ Neurosurg Sci 56:323-340, 2012

14. De Ridder D, Vanneste S, Plazier M, Menovsky T, van de Heyning P, Kovacs S, et al: Dorsolateral prefrontal cortex transcranial magnetic stimulation and electrode implant for intractable tinnitus. World Neurosurg 77:778-784, 2012

15. De Ridder D, Vanneste S, Plazier M, van der Loo E, Menovsky T: Burst spinal cord stimulation: toward paresthesia-free pain suppression. Neurosurgery 66:986-990, 2010

16. De Ridder D, Vanneste S, van der Loo E, Plazier M, Menovsky T, van de Heyning P: Burst stimulation of the auditory cortex: a new form of neurostimulation for noise-like tinnitus suppression. J Neurosurg 112:1289-1294, 2010 
17. De Ridder D, Vanneste S, Weisz N, Londero A, Schlee W, Elgoyhen AB, et al: An integrative model of auditory phantom perception: tinnitus as a unified percept of interacting separable subnetworks. Neurosci Biobehav Rev 44:16-32, 2014

18. De Ridder D, Verstraeten E, Van der Kelen K, De Mulder G, Sunaert S, Verlooy J, et al: Transcranial magnetic stimulation for tinnitus: influence of tinnitus duration on stimulation parameter choice and maximal tinnitus suppression. Otol Neurotol 26:616-619, 2005

19. Goebel G, Hiller W: [The tinnitus questionnaire. A standard instrument for grading the degree of tinnitus. Results of a multicenter study with the tinnitus questionnaire.] HNO 42:166-172, 1994 (Ger)

20. Jastreboff PJ: Phantom auditory perception (tinnitus): mechanisms of generation and perception. Neurosci Res 8:221254,1990

21. Joos K, Vanneste S, De Ridder D: Disentangling depression and distress networks in the tinnitus brain. PLoS One 7:e40544, 2012

22. Landgrebe M, Langguth B, Rosengarth K, Braun S, Koch A, Kleinjung T, et al: Structural brain changes in tinnitus: grey matter decrease in auditory and non-auditory brain areas. Neuroimage 46:213-218, 2009

23. Langguth B, Kreuzer PM, Kleinjung T, De Ridder D: Tinnitus: causes and clinical management. Lancet Neurol 12:920-930, 2013

24. Langguth B, Schecklmann M, Lehner A, Landgrebe M, Poeppl TB, Kreuzer PM, et al: Neuroimaging and neuromodulation: complementary approaches for identifying the neuronal correlates of tinnitus. Front Syst Neurosci 6:15, 2012

25. Lipsman N, Woodside DB, Giacobbe P, Hamani C, Carter JC, Norwood SJ, et al: Subcallosal cingulate deep brain stimulation for treatment-refractory anorexia nervosa: a phase 1 pilot trial. Lancet 381:1361-1370, 2013

26. Maudoux A, Lefebvre P, Cabay JE, Demertzi A, Vanhaudenhuyse A, Laureys S, et al: Auditory resting-state network connectivity in tinnitus: a functional MRI study. PLoS One 7:e36222, 2012

27. Maudoux A, Lefebvre P, Cabay JE, Demertzi A, Vanhaudenhuyse A, Laureys S, et al: Connectivity graph analysis of the auditory resting state network in tinnitus. Brain Res 1485:10-21, 2012

28. Mayberg HS, Lozano AM, Voon V, McNeely HE, Seminowicz D, Hamani C, et al: Deep brain stimulation for treatmentresistant depression. Neuron 45:651-660, 2005

29. Meeus O, De Ridder D, Van de Heyning P: Administration of the combination clonazepam-Deanxit as treatment for tinnitus. Otol Neurotol 32:701-709, 2011

30. Norena A, Micheyl C, Chéry-Croze S, Collet L: Psychoacoustic characterization of the tinnitus spectrum: implications for the underlying mechanisms of tinnitus. Audiol Neurootol 7:358-369, 2002

31. Noreña AJ, Eggermont JJ: Enriched acoustic environment after noise trauma abolishes neural signs of tinnitus. Neuroreport 17:559-563, 2006

32. Nowé V, Van de Heyning P, Parizel PM: MRI in patients with otovestibular complaints of unknown origin. B-ENT 3 (Suppl 7):27-35, 2007

33. Pascual-Marqui R: Discrete, 3D distributed, linear imaging methods of electric neuronal activity. Part 1: exact, zero error localization. (http://arxiv.org/abs/0710.3341) [Accessed June 25, 2015]

34. Pascual-Marqui R: Instantaneous and lagged measurements of linear and nonlinear dependence between groups of multivariate time series: frequency decomposition. (http://arxiv. org/abs/0711.1455) [Accessed June 25, 2015]

35. Pascual-Marqui RD: Standardized low-resolution brain electromagnetic tomography (sLORETA): technical details.
Methods Find Exp Clin Pharmacol 24 (Suppl D):5-12, 2002

36. Sadaghiani S, Hesselmann G, Kleinschmidt A: Distributed and antagonistic contributions of ongoing activity fluctuations to auditory stimulus detection. J Neurosci 29:13410 13417, 2009

37. Schecklmann M, Landgrebe M, Poeppl TB, Kreuzer P, Manner P, Marienhagen J, et al: Neural correlates of tinnitus duration and distress: A positron emission tomography study. Hum Brain Mapp 34:233-240, 2013

38. Schlee W, Hartmann T, Langguth B, Weisz N: Abnormal resting-state cortical coupling in chronic tinnitus. BMC Neurosci 10:11, 2009

39. Schlee W, Weisz N, Bertrand O, Hartmann T, Elbert T: Using auditory steady state responses to outline the functional connectivity in the tinnitus brain. PLoS One 3:e3720, 2008

40. Seeley WW, Menon V, Schatzberg AF, Keller J, Glover GH, Kenna H, et al: Dissociable intrinsic connectivity networks for salience processing and executive control. J Neurosci 27:2349-2356, 2007

41. Smits M, Kovacs S, de Ridder D, Peeters RR, van Hecke P, Sunaert S: Lateralization of functional magnetic resonance imaging (fMRI) activation in the auditory pathway of patients with lateralized tinnitus. Neuroradiology 49:669-679, 2007

42. Song JJ, De Ridder D, Van de Heyning P, Vanneste S: Mapping tinnitus-related brain activation: an activation-likelihood estimation metaanalysis of PET studies. J Nucl Med 53:1550-1557, 2012

43. van der Loo E, Congedo M, Vanneste S, Van De Heyning P, De Ridder D: Insular lateralization in tinnitus distress. Auton Neurosci 165:191-194, 2011

44. van der Loo E, Gais S, Congedo M, Vanneste S, Plazier M, Menovsky T, et al: Tinnitus intensity dependent gamma oscillations of the contralateral auditory cortex. PLoS One 4:e7396, 2009

45. Vanneste S, Azevedo A, De Ridder D: The effect of naltrexone on the perception and distress in tinnitus: an open-label pilot study. Int J Clin Pharmacol Ther 51:5-11, 2013

46. Vanneste S, Congedo M, De Ridder D: Pinpointing a highly specific pathological functional connection that turns phantom sound into distress. Cereb Cortex 24:2268-2282, 2013

47. Vanneste S, Heyning PV, Ridder DD: Contralateral parahippocampal gamma-band activity determines noise-like tinnitus laterality: a region of interest analysis. Neuroscience 199:481-490, 2011

48. Vanneste S, De Ridder D: The auditory and non-auditory brain areas involved in tinnitus. An emergent property of multiple parallel overlapping subnetworks. Front Syst Neurosci 6:31, 2012

49. Vanneste S, De Ridder D: Bifrontal transcranial direct current stimulation modulates tinnitus intensity and tinnitusdistress-related brain activity. Eur J Neurosci 34:605-614, 2011

50. Vanneste S, De Ridder D: Brain areas controlling heart rate variability in tinnitus and tinnitus-related distress. PLoS One 8:e59728, 2013

51. Vanneste S, De Ridder D: Differences between a single session and repeated sessions of $1 \mathrm{~Hz}$ TMS by double-cone coil prefrontal stimulation for the improvement of tinnitus. Brain Stimul 6:155-159, 2013

52. Vanneste S, Figueiredo R, De Ridder D: Treatment of tinnitus with cyclobenzaprine: an open-label study. Int J Clin Pharmacol Ther 50:338-344, 2012

53. Vanneste S, Ost J, Langguth B, De Ridder D: TMS by double-cone coil prefrontal stimulation for medication resistant chronic depression: a case report. Neurocase 20:61-68, 2014

54. Vanneste S, Plazier M, Van de Heyning P, De Ridder D: Repetitive transcranial magnetic stimulation frequency de- 
pendent tinnitus improvement by double cone coil prefrontal stimulation. J Neurol Neurosurg Psychiatry 82:1160-1164, 2011

55. Vanneste S, Plazier M, Van de Heyning P, De Ridder D: Transcutaneous electrical nerve stimulation (TENS) of upper cervical nerve (C2) for the treatment of somatic tinnitus. Exp Brain Res 204:283-287, 2010

56. Vanneste S, Plazier M, der Loo Ev, de Heyning PV, Congedo M, De Ridder D: The neural correlates of tinnitus-related distress. Neuroimage 52:470-480, 2010

57. Vanneste S, van de Heyning P, De Ridder D: The neural network of phantom sound changes over time: a comparison between recent-onset and chronic tinnitus patients. Eur J Neurosci 34:718-731, 2011

58. Vanneste S, van Dongen M, De Vree B, Hiseni S, van der Velden E, Strydis C, et al: Does enriched acoustic environment in humans abolish chronic tinnitus clinically and electrophysiologically? A double blind placebo controlled study. Hear Res 296:141-148, 2013

59. Weisz N, Hartmann T, Dohrmann K, Schlee W, Norena A: High-frequency tinnitus without hearing loss does not mean absence of deafferentation. Hear Res 222:108-114, 2006

\section{Disclosure}

Dr. De Ridder is a consultant for, holds a patent with, has received clinical or research support for the study described, and has received support for non-study-related clinical or research effort from St. Jude Medical.

\section{Author Contributions}

Conception and design: Vanneste. Acquisition of data: De Ridder, Joos. Analysis and interpretation of data: Vanneste. Drafting the article: De Ridder, Vanneste. Critically revising the article: De Ridder. Reviewed submitted version of manuscript: De Ridder, Joos. Statistical analysis: Vanneste. Administrative/technical/ material support: Joos. Study supervision: De Ridder.

\section{Correspondence}

Dirk De Ridder, Department of Surgical Sciences, Section of Neurosurgery, Dunedin School of Medicine, University of Otago, P.O. Box 56, Leith St., Dunedin 9054, New Zealand. email: dirk. deridder@otago.ac.nz. 